UF-IFT-HEP-99-6

Original: June 1997

Revised: June 1999

\title{
ELECTROWEAK THEORY WITHOUT HIGGS BOSONS
}

\author{
Angus F. Nicholson] \\ Department of Theoretical Physics, IAS \\ The Australian National University, Canberra, ACT 0200, Australia \\ Dallas C. Kennedy巴 \\ Department of Physics, University of Florida, \\ Gainesville, Florida 32611, USA
}

\begin{abstract}
A perturbative $S U(2)_{L} \times U(1)_{Y}$ electroweak theory containing $W, Z$, photon, ghost, lepton and quark fields, but no Higgs or other fields, gives masses to $W, Z$ and the non-neutrino fermions by means of an unconventional choice for the unperturbed Lagrangian and a novel method of renormalisation. The renormalisation extends to all orders. The masses emerge on renormalisation to one loop. To one loop the neutrinos are massless, the $A \leftrightarrow Z$ transition drops out of the theory, the $d$ quark is unstable and $S$-matrix elements are independent of the gauge parameter $\xi$.
\end{abstract}

International Journal of Modern Physics A 15 (2000) 1487

\footnotetext{
${ }^{1}$ Deceased

${ }^{2}$ e-mail: kennedy@phys.ufl.edu
} 


\section{Introduction}

It is widely considered that the Standard Model may be a low-energy effective field theory and that the Higgs boson might not exist (see e.g. [1,2]). This paper describes a perturbative $S U(2)_{L} \times U(1)_{Y}$ electroweak theory that contains only $W$ and $Z$ bosons, the photon, ghosts and $n$ generations of leptons and quarks, and in which $W, Z$ and fermions gain masses via renormalisation, in a way illustrated by a toy model [3]. There are no scalar fields, nor any new particles such as technifermions or preons, in the theory.

The effective Lagrangian density $\mathcal{L}$, given by (6) (section 2), is similar to that of standard electroweak (GSW) theory with its Higgs sector omitted; also, for simplicity, family mixing has been suppressed. The action is $S U(2)_{L} \times U(1)_{Y}$ BRS-invariant, as it is in GSW theory. We make the decomposition $\mathcal{L}=\mathcal{L}_{0}+\mathcal{L}_{1}$ and renormalise in an unconventional way. The physical masses of $W, Z$ and the fermions emerge on renormalisation to one loop. The theory is renormalisable, by the method proposed, to all orders.

Renormalising to one loop, we are able to choose values for the counterterm parameters that give masses to the $W$ and $Z$ bosons while keeping the photons massless, give suitable masses to the fermions, renormalise the vertices to their usual forms (with renormalised parameters $g_{R}, \theta_{R}$ that equal the unrenormalised quantities $g, \theta)$ and satisfy the conditions for $S U(2)_{L} \times U(1)_{Y}$ invariance. This renormalisation requires the neutrinos to be massless and $m_{W}=m_{Z} \cos \theta$ to hold, where $\theta$ is the Weinberg angle transforming from $W_{\mu}^{3}, B_{\mu}$ to $Z_{\mu}, A_{\mu}$ fields. The resulting propagators and vertices, and so $S$-matrix elements, are independent of the gauge parameter $\xi$ in $\mathcal{L}$. Also, the loop quantity $\pi_{\mu \nu}^{A Z}$ that mediates $A \leftrightarrow Z$ is finite, with the result that the divergent renormalisation factors $Z_{3}^{1 / 2}, Z_{3}^{1 / 2}$ from the contiguous $A, Z$ lines cause the $A \leftrightarrow Z$ transition to vanish from the theory, at least to one loop. Further, we do not need to renormalise the left and right components of fermion propagators separately.

In addition to this one-loop development, we outline, for the $W, Z$, photon and charged lepton propagators, and (as an example) the $W W Z$ vertex, proposed procedures for renormalising the theory to all orders, while continuing to fix arbitrary boson and fermion masses.

How should $\mathcal{L}_{0}$ be chosen? The norm is to take the quadratic part of a given $\mathcal{L}$ (prior to the insertion of any $Z_{i}=1-c_{i}$ renormalisation factors) to be $\mathcal{L}_{0}$. Thus, for

$$
\tilde{\mathcal{L}}=-\frac{1}{4}\left(\partial_{\mu} A_{\nu}-\partial_{\nu} A_{\mu}\right)^{2}+\bar{\psi}(i \not \partial-e A) \psi
$$


traditionally one would take for $\tilde{\mathcal{L}}_{0}$ the quadratic part explicitly present here; then $\tilde{\mathcal{L}}$, with a gauge-fixing term added and $Z_{i}$ factors inserted, would define a massless QED theory. However, we recall that Schwinger [4] gave an exact solution from (1) in $1+1$ spacetime dimensions, in which the only physical particle is a massive vector boson (see [5]). Also, we recall that $\mathcal{L}$ in GSW theory, in its initial form, does not contain vector boson mass terms of the form $M^{2} A_{\mu} A^{\mu}$, yet that theory gives massive $W$ and $Z$ bosons. From a contemporary perspective, in which $\mathcal{L}$ and its symmetries are central, it appears to be legitimate, given $\mathcal{L}$, to admit any choice for $\mathcal{L}_{0}$ that leads to a theory that is self-consistent and, in the case of a physical theory, fits experiment. As regards gauge symmetry, i.e. the BRS symmetry of the action, we recall that in QED, QCD and GSW theory, while the action $S=\int d^{4} x \mathcal{L}$ is invariant under the appropriate BRS transformations, the partial-actions $S_{0,1}=\int d^{4} x \mathcal{L}_{0,1}$ are not, so that gauge invariance places no immediate condition on the choice of $\mathcal{L}_{0}$; ultimately, that choice must lead to a gauge-independent $S$-matrix. $\mathrm{As}$ in $\mathrm{QED}$ and $\mathrm{QCD}$, the gauge symmetry is broken in the present theory in going from $\mathcal{L}$ to $\mathcal{L}_{0}$; however, we find that $S$-matrix elements are independent of the value of the gauge parameter $\xi$ in $\mathcal{L},(6)$, as is the case in $\mathrm{QED}, \mathrm{QCD}$.

We make a decomposition, $\mathcal{L}=\mathcal{L}_{0}+\mathcal{L}_{1}$, in which $\mathcal{L}_{0}$ contains fermion mass terms. Using the fields defined in section 2 , we place the mass term

$$
\mathcal{L}_{m}=-\sum\left(m_{e j 1} \bar{e}_{j} e_{j}+m_{u j 1} \bar{u}_{j \alpha} u_{j \alpha}+m_{d j 1} \bar{d}_{j \alpha} d_{j \alpha}\right)
$$

in $\mathcal{L}_{0}$ and place $-\mathcal{L}_{m}$ in $\mathcal{L}_{1}$, so that $\mathcal{L}$ is unchanged and the $S U(2)_{L} \times U(1)_{Y}$ invariance is unbroken. With the usual definition of "bare", the bare masses of all particles are zero, since there are no masses in $\mathcal{L}$. We refer to the masses in (2) as "initial" masses. For a stable fermion, the initial mass $m_{1}$ is later put equal to the renormalised mass $m_{R}$. The theory does not predict or impose values for the masses of non-neutrino fermions (or of $W$, $Z$ ) and in that sense the masses $m_{1}$ are arbitrary. This arbitrariness may be compared with that of the Yukawa coupling parameters that determine the fermion masses in GSW theory. The splitting of zero made to place $\mathcal{L}_{m},-\mathcal{L}_{m}$ in $\mathcal{L}_{0}, \mathcal{L}_{1}$ is logically on the same footing as the splitting made of $m \bar{\psi} \psi$ in QED, to place $m_{\text {phys }} \bar{\psi} \psi$ in $\mathcal{L}_{0}$ and $\delta m \bar{\psi} \psi$ in $\mathcal{L}_{1}$. It might appear that the $\mathcal{L}_{m},-\mathcal{L}_{m}$ step must be nugatory, since resulting mass terms from $\mathcal{L}_{0}, \mathcal{L}_{1}$ cancel in the denominators of the full, improper propagators, i.e. in $\left\{\not p-m-\left[-m+\sum(\not p)+c . t.\right]\right\}$; however, the $m_{j 1}$ also contribute to the self-energy functions $\sum(\not p)$ and $\pi_{\mu \nu}(k)$ for the fermions and $W, Z$ bosons, leading to masses for these particles. 
The self-energy tensor for $W$ or $Z$ can be written in the form

$$
\pi_{\mu \nu}(k)=\left(k_{\mu} k_{\nu}-g_{\mu \nu} k^{2}\right) \pi\left(k^{2}\right)+k_{\mu} k_{\nu} \tau\left(k^{2}\right)+g_{\mu \nu} \rho\left(k^{2}\right)
$$

with $\rho\left(k^{2}\right)$ defined not to contain a factor $k^{2}$. The terms $\rho_{W}\left(k^{2}\right), \rho_{Z}\left(k^{2}\right)$ are central to the generation of the $W$ and $Z$ masses in this theory. To one loop, only fermion loops contribute to $\rho\left(k^{2}\right)$, and $\tau\left(k^{2}\right)$ is zero. The development requires that the initial fermion masses $m_{j 1}$ are of $O\left(g^{k}\right), k \geq 1$, and we take $k=1$ in this paper. Then it turns out that the $W$ and $Z$ masses are $O(g)$, as they are in GSW theory.

We use dimensional regularisation (which is usual in electroweak theory $[6,7]$ ), working in $d=4-2 \epsilon$ dimensions. Much work has been done on the problem of $\gamma^{5}$ in this regularisation and its variant, dimensional reduction, and the various approaches have given the same results in a variety of calculations to two and three loops (see e.g. [8-14]). Using the prescription of a formal $\gamma^{5}$ that is totally anticommuting with $\gamma^{\mu}$ in $d$ dimensions [15], and the couplings and propagators given in section 2 , a calculation to one loop of the divergent parts of $\rho_{W}\left(k^{2}\right)$ and $\rho_{Z}\left(k^{2}\right)$ gives

$$
\rho_{W \epsilon}^{(2)}=\rho_{Z \epsilon}^{(2)} \cos ^{2} \theta=\frac{g^{2}}{32 \pi^{2} \epsilon} \sum\left(m_{e j 1}^{2}+3 m_{u j 1}^{2}+3 m_{d j 1}^{2}\right) .
$$

Since the $S U(2)_{L} \times U(1)_{Y}$ invariance does not permit $\mathcal{L},(6)$, to contain gauge boson mass counterterms, these terms cannot be directly cancelled. In our renormalisation procedure we use the $c_{W}, c_{B}$ counterterm parameters in $\mathcal{L}$ to cancel the most divergent part (at any given order) of $k^{2} \pi_{W}\left(k^{2}\right)-\rho_{W}\left(k^{2}\right)$ at $k^{2}=m_{W}^{2}$, and similarly for $Z$.

The $W$ and $Z$ bosons and many of the fermions are unstable; however, the usual perturbative and LSZ formalisms [16] do not accommodate unstable particles in a consistent way (see e.g. [17-19]). For example, in the usual approach the initial and final state vectors $|i\rangle,|f\rangle$ of a matrix element $S_{f i}$ represent physical states that at $t \rightarrow \pm \infty$ comprise specified sets of physical particles assumed stable. Consequently, the well-known unitarity argument for the existence of Higgs bosons [20], involving $W$ or $Z$ bosons in initial or final states, is open to question. It is often assumed that stable-particle perturbation theory can be used with an unstable particle represented by a propagator in which the mass is complex, i.e. that the (principal) denominator of the propagator is of the form $k^{2}-\mathcal{M}^{2}$, with $\mathcal{M}^{2}$ complex (see e.g. $[21,22]$ for the complex mass of $Z$ in GSW theory). Then, if we apply the LSZ reduction formula [7] to a diagram containing an unstable particle 
or an external line, the result is zero, since the projection operator for the particle, containing a real mass $m$ (from $\mathcal{L}_{0}$ ), operates on the propagator, containing a complex mass $\mathcal{M}$. Since the zero result is independent of $m$, the reduction formula does not restrict $m$, as it does for a stable particle (to be $m_{R}$ ). On this basis, we take $m_{W 1}=m_{Z 1}=0$ in this paper, and renormalise to the complex masses $\mathcal{M}_{W}, \mathcal{M}_{Z}$. Further, we see that if we deal with unstable particles by means of complex-mass propagators, then the usual interpretation by means of the Cutkosky rules [24] of the $S$-matrix unitarity relation, which, writing $S=1+i T$, is

$$
T_{f i}-T_{i f}^{*}-i \sum T_{n f}^{*} T_{n i}=0,
$$

is rendered ambiguous, because for a complex-mass unstable particle one cannot identify a state carrying a real mass corresponds to the physical particle. We propose that the physical interpretation should be made at the level of the renormalised theory. These issues are problems also for the Standard Model. Questions of gauge invariance and unitarity with unstable particles, and the complex $Z$ pole mass, are discussed in the literature [2527]. In this paper we use the standard perturbative formalism even though most of the particles are unstable, as is commonly done for the Standard Model, but it is clear that we cannot deal with unitarity in the absence of a consistent unstable-particle extension of perturbation theory. Consequently, we do not discuss unitarity further in this paper.

Section 2 deals with the Lagrangian, the $S U(2)_{L} \times U(1)_{Y}$ BRS-invariance of the action, $\mathcal{L}_{0}$, quantisation and propagators. Section 3 discusses the renormalisation to all orders of the $W$ and $Z$ propagators to obtain $W$ and $Z$ masses, and of the photon and ghost propagators; then renormalises these propagators to one loop explicitly; also, it shows how $A \leftrightarrow Z$ mixing drops out of the theory to one loop. In section 4 the renormalisation of charged lepton propagators to all orders is discussed, followed by the renormalisation of all lepton and quark propagators to one loop, to give suitable masses. In section 5 we deal with the vertices. We outline a proposed renormalisation of the $W W Z$ vertex to all orders, then complete the renormalisation of the theory to one loop, showing that the resulting counterterm parameters satisfy the conditions for $S U(2)_{L} \times U(1)_{Y}$ invariance. We also point out that the theory renormalised to one loop has $S$-matrix elements that are independent of the gauge parameter $\xi$. Section 6 sums up these themes and their relation to other, similar work. 


\section{Lagrangian, $S U(2)_{L} \times U(1)_{Y}$ invariance, $\mathcal{L}_{0}$, propagators}

The Lagrangian density is essentially that of standard GSW theory with Higgs fields omitted and quark mixing suppressed. It is

$$
\begin{aligned}
\mathcal{L}= & \left.-\frac{1}{4} f_{W} W_{\mu \nu}^{k} W_{k}^{\mu \nu}-\frac{1}{4} f_{B} B_{\mu \nu} B^{\mu \nu}-f_{6}(2 \xi)^{-1}\left[(\partial \cdot W)^{2}+(\partial \cdot B)^{2}\right)\right] \\
& +\frac{1}{2} f_{1} g \epsilon^{i j k} W_{\mu \nu}^{i} W_{j}^{\mu} W_{k}^{\nu}-\frac{1}{4} f_{5} g^{2} \epsilon_{i j k} \epsilon^{i \ell n} W_{\mu}^{j} W_{\nu}^{k} W_{\ell}^{\mu} W_{n}^{\nu} \\
& +\tilde{f}_{3}\left(\partial_{\mu} \eta_{k}^{*}\right) \partial^{\mu} \eta_{k}+\tilde{f}_{1} g \epsilon^{i j k}\left(\partial_{\mu} \eta_{i}^{*}\right) \eta_{j} W_{k}^{\mu}+\tilde{f}_{3}\left(\partial_{\mu} \eta_{B}^{*}\right) \partial^{\mu} \eta_{B} \\
& +i \sum\left[\begin{array}{c}
f_{2 \ell L} \bar{\ell}_{L} \not \partial \ell_{L}+f_{2 e R} \bar{e}_{R} \not \partial e_{R}+f_{2 q L} \bar{q}_{\alpha L} \not \partial q_{\alpha L} \\
+f_{2 u R} \bar{u}_{\alpha R} \not u_{\alpha R}+f_{2 d R} \bar{d}_{\alpha R} \not d_{\alpha R}
\end{array}\right] \\
& -\sum\left\{\begin{array}{c}
f_{1 \ell L} \bar{\ell}_{L}\left(\frac{1}{2} g \tau \cdot W\right) \ell_{L}+\left(c_{\ell L}-\frac{1}{2} g^{\prime}\right) \bar{\ell}_{L} \not B \ell_{L}+\left(c_{e R}-g^{\prime}\right) \bar{e}_{R} \not B e_{R} \\
+f_{1 q L} \bar{q}_{\alpha L}\left(\frac{1}{2} g \tau \cdot W\right) q_{\alpha L}+\left(c_{q L}+\frac{1}{6} g^{\prime}\right) \bar{q}_{\alpha L} \not B q_{\alpha L} \\
+\left(c_{u R}+\frac{2}{3} g^{\prime}\right) \bar{u}_{\alpha R} \not B u_{\alpha R}+\left(c_{d R}-\frac{1}{3} g^{\prime}\right) \bar{d}_{\alpha R} \not B d_{\alpha R}
\end{array}\right\},
\end{aligned}
$$

where $g^{\prime}=g \tan \theta, f_{i}=1-c_{i}$, with the $c_{i}\left(c_{W}, c_{B}, \ldots, c_{2 \ell L}, \ldots\right)$ being counterterm parameters of $O\left(g^{2}\right)$ and higher; $W_{\mu \nu}^{k} \equiv \partial_{\mu} W_{\nu}^{k}-\partial_{\nu} W_{\mu}^{k}(k=1,2,3)$ and similarly for $B_{\mu \nu}$ and for $A_{\mu \nu}, Z_{\mu \nu}$ below; and $\eta_{k}^{*}, \eta_{k}, \eta_{B}^{*}, \eta_{B}$ are the ghost fields. The sums are over $n$ generations of lepton and quark fields $\ell_{j}\left(\nu_{j}, e_{j}\right), q_{j \alpha}\left(u_{j \alpha}, d_{j \alpha}\right), j=1, \ldots, n$ and over the colour index $\alpha$; suppressing $j$, we have written $\ell_{L}, q_{\alpha L}$ for the left-handed doublets, $e_{R}$, $u_{\alpha R}, d_{\alpha R}$ for the singlets (the fields $\nu_{j R}$ are absent) and $f_{2 \ell L}, f_{2 e R}, \ldots$ for $f_{2 \ell j L}, f_{2 e j R}, \ldots, f_{1 \ell j L}$, etc. In $d=4-2 \epsilon$ dimensions, $g$ is to be replaced by $g \mu^{\epsilon}$, with $g$ dimensionless and $\mu$ a scale mass, in the usual way; this is left implicit in what follows.

It is straightforward to ensure in the usual way $[7,28]$ that we have an $S U(2)_{L} \times U(1)_{Y}$ gauge theory. We transform to bare fields $W_{\mu}^{k b}=$ $f_{W}^{1 / 2} W_{\mu}^{k}, \ldots$ and parameters $\xi_{W}^{b}=f_{6}^{-1} f_{W} \xi, \xi_{B}^{b}=f_{6}^{-1} f_{B} \xi, g_{1}^{b}=f_{1} f_{W}^{-3 / 2} g$, $g_{5}^{b}=f_{5}^{1 / 2} f_{W}^{-1} g, \ldots$ Then the action $S=\int d^{4} x \mathcal{L}$ is invariant under an $S U(2)_{L}$ BRS transformation [28] of the bare fields, provided that

$$
\frac{f_{W}}{f_{1}}=\frac{f_{1}}{f_{5}}=\frac{\tilde{f}_{3}}{\tilde{f}_{1}}=\frac{f_{2 \ell j L}}{f_{1 \ell j L}}=\frac{f_{2 q j L}}{f_{1 q j L}}
$$

which ensures that $g_{1}^{b}=g_{5}^{b}=\cdots$. The action is also invariant under a suitable $U(1)_{Y}$ BRS transformation of the fields without the imposition of any conditions, in the usual way.

Returning to the form (6), we transform in the standard way to the fields $A_{\mu}=W_{\mu}^{3} \sin \theta+B_{\mu} \cos \theta, Z_{\mu}=W_{\mu}^{3} \cos \theta-B_{\mu} \sin \theta, W_{\mu}^{ \pm}=(1 / \sqrt{2})\left(W_{\mu}^{1} \mp\right.$ 
$\left.i W \mu^{2}\right), \eta^{*+}=(1 / \sqrt{2})\left(\eta_{1}^{*}-i \eta_{2}^{*}\right), \eta^{-}=(1 / \sqrt{2})\left(\eta_{1}+i \eta_{2}\right), \eta^{*-}=(1 / \sqrt{2})\left(\eta_{1}^{*}+\right.$ $\left.i \eta_{2}^{*}\right), \eta^{+}=(1 / \sqrt{2})\left(\eta_{1}-i \eta_{2}\right), \eta^{* A}=\eta_{3}^{*} \sin \theta+\eta_{B}^{*} \cos \theta, \eta^{A}=\eta_{3} \sin \theta+\eta_{B} \cos \theta$, $\eta^{* Z}=\eta_{3}^{*} \cos \theta-\eta_{B}^{*} \sin \theta$ and $\eta^{Z}=\eta_{3} \cos \theta-\eta_{B} \sin \theta$. The resulting couplings of the $W, Z, A$, new ghost and fermion fields are the same as those in GSW theory [7]. The quadratic boson-ghost part of $\mathcal{L}$ is

$$
\begin{aligned}
\mathcal{L}_{Q}= & -\frac{1}{4}\left[f_{A} A_{\mu \nu} A^{\mu \nu}+f_{Z} Z_{\mu \nu} Z^{\mu \nu}+2 f_{W} W_{\mu \nu}^{+} W_{-}^{\mu \nu}\right]+\frac{1}{2} c_{A Z} A_{\mu \nu} Z^{\mu \nu} \\
& -f_{6}(2 \xi)^{-1}\left[(\partial \cdot A)^{2}+(\partial \cdot Z)^{2}+2\left(\partial \cdot W^{+}\right)\left(\partial \cdot W^{-}\right)\right] \\
& \tilde{f}_{3}\left[\left(\partial_{\mu} \eta^{*+}\right) \partial^{\mu} \eta^{-}+\left(\partial_{\mu} \eta^{*-}\right) \partial^{\mu} \eta^{+}+\left(\partial_{\mu} \eta^{* A}\right) \partial^{\mu} \eta^{A}+\left(\partial_{\mu} \eta^{* Z}\right) \partial^{\mu} \eta^{Z}\right],
\end{aligned}
$$

where

$$
\begin{gathered}
f_{A, Z}=f_{W, B} \sin ^{2} \theta+f_{B, W} \cos ^{2} \theta, \\
c_{A Z}=\left(c_{W}-c_{B}\right) \sin \theta \cos \theta .
\end{gathered}
$$

The counterterm parameters $c_{A}, c_{Z}$ are defined by $f_{A}=1-c_{A}, f_{Z}=1-c_{Z}$.

As discussed in section 1 , initial quark and charged lepton masses are introduced by placing $\mathcal{L}_{m},(2)$, in $\mathcal{L}_{0}$. We also place $-\mathcal{L}_{m}$ in $\mathcal{L}_{1}$, so that $\mathcal{L}$ is unchanged and the $S U(2)_{L} \times U(1)_{Y}$ invariance is not broken. In section 4 the masses $m_{e j 1}, m_{u j 1}, m_{d j 1}$ are taken to be $O(g)$ (and we find that the initial neutrino masses must be zero). This leads, in section 3, to $W$ and $Z$ masses of $O(g)$, which is the case also in GSW theory. Including $\mathcal{L}_{m}$, we take $\mathcal{L}_{0}$ to be

$$
\begin{aligned}
\mathcal{L}_{0}= & -\frac{1}{4}\left(A_{\mu \nu} A^{\mu \nu}+Z_{\mu \nu} Z^{\mu \nu}+2 W_{\mu \mu}^{+} W_{-}^{\mu \nu}\right) \\
& -(2 \xi)^{-1}\left[(\partial \cdot A)^{2}+(\partial \cdot Z)^{2}+2\left(\partial \cdot W^{+}\right)\left(\partial \cdot W^{-}\right)\right] \\
& +\left(\partial_{\mu} \eta^{*+}\right) \partial^{\mu} \eta^{-}+\left(\partial_{\mu} \eta^{*-} \partial^{\mu} \eta^{+}+\left(\partial_{\mu} \eta^{* A}\right) \partial^{\mu} \eta^{A}+\left(\partial_{\mu} \eta^{* Z}\right) \partial^{\mu} \eta^{Z}\right. \\
& +\sum\left[\bar{\ell}\left(i \not \partial-m_{\ell 1}\right) \ell+\bar{q}_{\alpha}\left(i \not \partial-m_{q 1}\right) q_{\alpha}\right]
\end{aligned}
$$

in which the fermion part has been abbreviated. If all the particles were stable, we could proceed by using the usual path integral or canonical quantisation method. We assume that perturbation theory can be extended to encompass unstable particles so that we obtain from $\mathcal{L}_{0}$ the propagators that would result in the stable-particle case, viz.

$$
\begin{aligned}
i D_{\mu \nu}^{W, Z, A}(k) & =-i k^{-2}\left[g_{\mu \nu}+(\xi-1) k_{\mu} k_{\nu} k^{-2}\right] \\
i \tilde{D}\left(k^{2}\right) & =i k^{-2} \\
i S_{F}(\not p) & =i\left(\not p-m_{j 1}\right)^{-1}
\end{aligned}
$$


for the massless proto- $W$, proto- $Z, A$, ghost and the fermion fields. Because we do not give a physical interpretation of the basis vectors of the state space (or protoparticles) generated by $W_{\mu}$ and $Z_{\mu}$, we have the simplification that the $W_{\mu}, Z_{\mu}$ part of $\mathcal{L}_{0}$ can be quantised without the imposition of a gauge condition designed to single out physical states. For the $A_{\mu}$ part of $\mathcal{L}_{0}$ we can proceed in a standard way. The usual quantisation procedure gives, from $\mathcal{L}_{0}$, a set of particle states that includes a unique, nondegenerate vacuum, like that of QED, in which the VEV of each field is zero.

With these propagators, and with the fermion generations and fermiongauge boson couplings the same as they are in GSW theory, there are no anomalies in the theory.

\section{Renormalised boson and ghost propagators and masses}

For each boson, labelled by $J=W, Z, A$, we sum the series

$$
i \mathcal{D}_{J \mu \nu}=i D_{J \mu \mu}+i D_{J \mu \sigma}\left(i \hat{\pi}_{J}^{\sigma \rho}\right) i D_{J \rho \nu}+\cdots,
$$

where $\hat{\pi}_{J}^{\sigma \rho}$ is defined by

$$
\hat{\pi}_{J \mu \nu}=\left(k_{\mu} k_{\nu}-g_{\mu \nu} k^{2}\right) \hat{\pi}_{J}\left(k^{2}\right)+k_{\mu} k_{\nu} \hat{\tau}\left(k^{2}\right)+g_{\mu \nu} \rho\left(k^{2}\right)
$$

in which

$$
\hat{\pi}_{J}\left(k^{2}\right)=\pi_{J}\left(k^{2}\right)-c_{J}, \quad \hat{\tau}_{J}\left(k^{2}\right)=\tau\left(k^{2}\right)+c_{6} \xi^{-1},
$$

where $\pi_{J}\left(k^{2}\right), \tau\left(k^{2}\right), \rho\left(k^{2}\right)$ are given by the self-energy integrals with $\rho\left(k^{2}\right)$ defined not to contain a factor $k^{2}$, and $c_{J}, c_{6}$ are counterterm parameters, with $c_{A}, c_{Z}$ given by (9). In $A \leftrightarrow Z$ subdiagrams the $A, Z$ lines are linked by $i \hat{\pi}_{\mu \nu}^{A Z}$, with

$$
\hat{\pi}_{\mu \nu}^{A Z}=\left(k_{\mu} k_{\nu}-g_{\mu \nu} k^{2}\right) \hat{\pi}_{A Z}\left(k^{2}\right)+k_{\mu} k_{\nu} \tau_{A Z}\left(k^{2}\right)+g_{\mu \nu} \rho_{A Z}\left(k^{2}\right),
$$

in which

$$
\hat{\pi}_{A Z}\left(k^{2}\right)=\pi_{A Z}\left(k^{2}\right)-c_{A Z},
$$

with $c_{A Z}$ given by (10). To one loop (see below), $\tau_{J}\left(k^{2}\right)=0$, the divergent parts of $\rho_{W}, \rho_{Z}$ are given by (4) and $\rho_{A}\left(k^{2}\right)=0$, which might be true to all orders, as it is in QED.

In the usual way, the sum in (15) is given, correctly to any chosen $O\left(g^{2 n}\right)$, by

$$
i \mathcal{D}_{J \mu \nu}=\frac{-i}{\left(1+\hat{\pi}_{J}\right) k^{2}-\rho_{J}}\left[g_{\mu \nu}+k_{\mu} k_{\nu} Q_{J}\left(k^{2}\right)\right],
$$


where

$$
Q_{J}\left(k^{2}\right)=\frac{\xi\left(\hat{\pi}_{J}+\hat{\tau}_{J}+1\right)-1}{k^{2}-\xi\left(\rho_{J}+k^{2} \hat{\tau}_{J}\right)} .
$$

We outline a proposed procedure for renormalising the $W$ and $Z$ propagators to all orders to give masses to these bosons, and renormalising the photon and ghost propagators to all orders. Then we renormalise the $W$, $Z$, photon and ghost propagators to one loop.

We define the $O\left(g^{2 n}\right)$ component $\pi_{2 n}\left(k^{2}\right)$ in $\pi\left(k^{2}\right)=\sum \pi_{2 n}\left(k^{2}\right)$, and, similarly, $\tau_{2 n}$ and $\rho_{2 n}$. Following the conventions of calculations beyond one loop in QED and QCD, in which the propagators have the forms (12), (13), (14), and the dimensions of coupling parameters and the topologies of diagrams are essentially the same as in this theory, we can take $\pi_{2 n}\left(k^{2}\right)$ (generated in the usual way by $n$-loop diagrams not containing counterterm insertions plus $(n-j)$-loop diagrams with insertions) in the form

$$
\pi_{2 n}\left(k^{2}\right)=g^{2 n}\left[\pi_{2 n, n} \epsilon^{-n}+\pi_{2 n, n-1}\left(k^{2}, m_{j 1}^{2}\right) \epsilon^{-n+1}+\cdots+\pi_{2 n, 0}\left(k^{2}, m_{j 1}^{2}\right)\right],
$$

where $\pi_{2 n, n}$ is real and independent of $k^{2}, g^{2}$ and the initial masses $m_{j 1}$. Since the $m_{j 1}$ are $O(g)$, and we encounter $\pi_{2 n}\left(k^{2}=\mathcal{M}^{2}\right)$ with $\mathcal{M}$ of $O(g)$, any expansions of $\pi_{2 n, \sigma}\left(k^{2}, m_{j 1}^{2}\right), \pi_{2 n, \sigma}\left(\mathcal{M}^{2}, m_{j 1}^{2}\right)$ as series in $g^{2}$ would cause $\pi_{2 n}\left(k^{2}\right)$ to contain $g^{2 N}, N>n$. However, we do not need to make such expansions in order to renormalise the theory, and it is convenient to refer to $\pi_{2 n}\left(k^{2}\right), \pi_{2 n}\left(\mathcal{M}^{2}\right)$ as being of $O\left(E g^{2 n}, \epsilon^{-n}\right)$, where $E g^{2 n}$ indicates the explicit $g^{2 n}$ factor in (22) and $\epsilon^{-n}$ is the highest power of $\epsilon^{-1}$ that is present. (This factor $\epsilon^{-n}$ is independent of any expansion that might be made of $\pi_{2 n, \sigma}$ terms as series in $g^{2}$. When we encounter $g^{\sigma} \pi_{2 n}$ terms we shall extend the notation to $O\left(E g^{2 n+\sigma}, \epsilon^{-n}\right)$.) We treat $\tau\left(k^{2}\right)$ in the same way. On dimensional grounds and from the structure of the integrals, $\rho\left(k^{2}\right)$ is of the form $\sum m_{j 1}^{2} \rho_{j}\left(k^{2}\right)$, with $m_{j 1}=\beta_{j 1} g$ (section 4$)$, so that an $(n-2)$-loop diagram free of counterterm insertions generates a component of $\rho\left(k^{2}\right)$ of the form (22), with $\rho_{2 n, n}=\rho_{2 n, n}\left(\beta_{j 1}^{2}\right)$, of $O\left(E g^{2 n} \epsilon^{-n}\right)$, real and independent of $k^{2}$ and $g^{2}$. Consequently, an $n$-loop diagram generates terms $\pi_{2 n}\left(k^{2}\right)$, $\rho_{2 n+2}\left(k^{2}\right)$ of $O\left(E g^{2 n}, \epsilon^{-n}\right), O\left(E g^{2 n+2}, \epsilon^{-n}\right)$. To $N$ loops (summing over appropriate lower-order diagrams up to $N$-loop diagrams free of counterterm insertions) we have the quantities $\pi_{(N)}\left(k^{2}\right), \rho_{(N)}\left(k^{2}\right)$, of $O\left(E g^{2 N}, \epsilon^{-N}\right)$, $O\left(E g^{2 N+2}, \epsilon^{-N}\right)$. Writing $\left(\partial / \partial k^{2}\right) \pi\left(k^{2}\right)=\pi^{\prime}\left(k^{2}\right)$, etc., we see from (22) that

$$
\frac{\pi_{(N)}^{\prime}\left(k^{2}\right)}{\pi_{(N)}\left(k^{2}\right)} \rightarrow 0, \quad \frac{\pi_{(N)}^{\prime \prime}\left(k^{2}\right)}{\pi_{(N)}\left(k^{2}\right)} \rightarrow 0, \quad \ldots
$$


as $\epsilon \rightarrow 0$, and similarly for $\pi_{2 n}^{\prime}, \pi_{2 n}$, and $\rho^{\prime}, \rho$, etc.

It is sufficient for $c_{J}$ to be of the form

$$
c_{J}=\sum c_{J, 2 n} g^{2 n} \epsilon^{-n},
$$

i.e. that the counterterm parameters $c_{W}, c_{Z}, c_{A}$ need not contain components of order $g^{2 n} \epsilon^{-\sigma}, \sigma<n$. We define, for $J=W, Z$,

$$
\begin{gathered}
\hat{\pi}_{J N L}=\sum_{n=1}^{N}\left(\pi_{J, 2 n, n}-c_{J, 2 n}\right) g^{2 n} \epsilon^{-n}, \\
\rho_{J N L}=\sum_{n=1}^{N} \rho_{J, 2 n+2, n} g^{2 n+2} \epsilon^{-n},
\end{gathered}
$$

which are independent of $k^{2}$ and real. Clearly $\hat{\pi}_{J N L}, \rho_{J N L}$ are the leading divergent parts of $\left(\pi_{J}\left(k^{2}\right)-c_{J}\right), \rho_{J}\left(k^{2}\right)$ taken to $N$ loops. They are of $O\left(g^{2 N} \epsilon^{-N}\right), O\left(g^{2 N+2} \epsilon^{-N}\right)$ (which we rely on below; they are also $O\left(E g^{2 N}, \epsilon^{-N}\right)$, $\left.O\left(E g^{2 N+2}, \epsilon^{-N}\right)\right)$.

To $N$ loops, and for $J=W, Z$, the principal denominator in $\mathcal{D}_{J \mu \nu},(20)$ is easily seen to be

$$
d_{J N}\left(k^{2}\right)=\left(1+\hat{\pi}_{J N L}\right) k^{2}-\rho_{J N L}+R_{J N}\left(k^{2}\right)_{(2 N,-N+1)},
$$

where the $(2 N,-N+1)$ subscript indicates that $R_{J N}\left(k^{2}\right)$ is of $O\left(E g^{2 N}, \epsilon^{-N+1}\right)$. For the derivatives of $d_{J N}\left(k^{2}\right)$ we then have

$$
\begin{gathered}
d_{J N}^{\prime}\left(k^{2}\right)=1+\hat{\pi}_{J N L}+R_{J N}^{\prime}\left(k^{2}\right)_{(2 N,-N+1)}, \\
d_{J N}^{(\sigma)}\left(k^{2}\right)=R_{J N}^{(\sigma)}\left(k^{2}\right)_{(2 N,-N+1)},
\end{gathered}
$$

where $(\sigma)$ indicates any derivative beyond the first.

We renormalise the $W$ and $Z$ propagators to have the (principal) pole masses $\mathcal{M}_{W}, \mathcal{M}_{Z}$, with

$$
\mathcal{M}_{J}^{2}=m_{J}^{2}-i \delta_{J} \quad(J=W, Z)
$$

where

$$
m_{J}=\beta_{J} g, \quad \delta_{J}=\delta_{J 4} g^{4}+\delta_{J 6} g^{6}+\cdots
$$

and $m_{J}, \delta_{J}$ are arbitrary (to be fitted to experiment in a complete physical theory). (As usual, the renormalisation of the $W, Z$ and photon propagators is to proceed, order by order, in step with the order by order renormalisation 
of ghost and fermion propagators and vertices as discussed below, to fix, at each order, the counterterms needed for renormalisation at higher orders.) We write

$$
d_{J N}\left(k^{2}\right)=d_{J N}\left(\mathcal{M}^{2}\right)+d_{J N}^{\prime}\left(\mathcal{M}^{2}\right)\left(k^{2}-\mathcal{M}_{J}^{2}\right)+\frac{1}{2 !} d_{J N}^{\prime \prime}\left(\mathcal{M}^{2}\right)\left(k^{2}-\mathcal{M}_{J}^{2}\right)^{2}+\cdots
$$

From (27) we obtain

$$
\begin{aligned}
d_{J N}\left(\mathcal{M}^{2}\right)=\left(1+\hat{\pi}_{J N L}\right) m_{J}^{2} & -\left[1+\hat{\pi}_{J(N-1) L}\right] i \delta+O\left(E g^{2 N+4}, \epsilon^{-N}\right) \\
& -\rho_{J N L}+R_{J N}\left(k^{2}\right)_{(2 N,-N+1)}
\end{aligned}
$$

We impose the mass condition

$$
\hat{\pi}_{J N L} m_{J}^{2}-\rho_{J N L}=0
$$

at $N=1,2, \ldots$ loops; i.e. at every order $n$ we take, using (25), (26), (31),

$$
c_{J, 2 n}=\pi_{J, 2 n, n}-\beta_{J}^{-2} \rho_{J, 2 n+2, n} .
$$

At each $O\left(g^{2 n}\right)$ we are cancelling the most divergent $\left(\epsilon^{-n}\right)$ part of the $g_{\mu \nu}\left[-k^{2} \pi\left(k^{2}\right)+\rho\left(k^{2}\right)\right]$ component of $\pi_{\mu \nu}(k)$, (3), at $k^{2}=m_{J}^{2}$. We may compare this with the usual subtraction procedures in QED and QCD, in which (with $\rho\left(k^{2}\right)=0$ ) the divergences in $\pi$ are fully cancelled, order by order, at every $O\left(g^{2 n} \epsilon^{-\sigma}\right), \sigma \leq n$. In this theory it is not necessary to cancel all the divergent parts of the $g_{\mu \nu}$ component of $\pi_{\mu \nu}$, as is shown in what follows.

We define

$$
\widehat{Z}_{J N}=\left(1+\hat{\pi}_{J N L}\right)^{-1},
$$

which turns out to be the renormalisation factor. We see from (33), (28), (29), using (30), (31), (25), (26) and (34), that, correctly to $O\left(g^{2 N+2}\right)\left(\hat{\pi}_{J N L}\right.$, $\rho_{J N L}$ are precisely $O\left(g^{2 N}\right), O\left(g^{2 N+2}\right)$ as well as $\left.O\left(E g^{2 N}\right), O\left(E g^{2 n+2}\right)\right)$,

$$
\begin{gathered}
\widehat{Z}_{J N} d_{J N}\left(\mathcal{M}^{2}\right)=O(\epsilon), \\
\widehat{Z}_{J N} d_{J N}^{\prime}\left(\mathcal{M}^{2}\right)=1+O(\epsilon),
\end{gathered}
$$

and

$$
\widehat{Z}_{J N} d_{J N}^{(\sigma)}\left(\mathcal{M}^{2}\right)=O(\epsilon)
$$

Then from (32) we obtain, correctly to $O\left(g^{2 N+2}\right)$,

$$
\lim _{\epsilon \rightarrow 0}\left[\widehat{Z}_{J N} d_{J N}\left(k^{2}\right)\right]=k^{2}-\mathcal{M}^{2}
$$


Since $N$ is arbitrary, this result extends to any order. Then from (20) we see that the renormalised propagator is

$$
i \mathcal{D}_{J \mu \nu}^{R}=\frac{-i}{k^{2}-\mathcal{M}_{J}^{2}}\left\{g_{\mu \nu}+k_{\mu} k_{\nu} \lim _{\epsilon \rightarrow 0}\left[Q_{J}\left(k^{2}\right)\right]\right\}
$$

for $J=W, Z$ (we deal with $Q_{J}\left(k^{2}\right)$ below), and that the renormalisation factor is, to $N$ loops, $\widehat{Z}_{J N}$.

For the photon we have the denominator

$$
d_{A}\left(k^{2}\right)=\left[1+\pi_{A}\left(k^{2}\right)-c_{A}\right] k^{2}-\rho_{A}\left(k^{2}\right) .
$$

We define

$$
\widehat{Z}_{A N}=\left(1+\hat{\pi}_{A N L}\right)^{-1}
$$

where $\hat{\pi}_{A N L}$, independent of $k^{2}$ and real, is defined by (25) with $J=A$, while $c_{A}$ is determined, by (9), from $c_{W}, c_{Z}$ given by (35); it is of the form of $c_{J},(24)$. To one loop, (55), (63) below show that $\rho_{A}\left(k^{2}\right)=0$ and that $\hat{\pi}_{A}$ diverges. If $\rho_{A}\left(k^{2}\right)=0$ to all orders, as holds true in QED, or if $\rho_{A}$ is less divergent than $\hat{\pi}_{A}$ at each order, then the photon remains massless. If that is so, we have

$$
d_{A N}\left(k^{2}\right)=\left(1+\hat{\pi}_{A N L}\right) k^{2}-\rho_{A N}\left(k^{2}\right)_{(2 N+2,-N+\sigma)}+O\left(E g^{2 N}, \epsilon^{-N+1}\right)
$$

and, correctly to $O\left(g^{2 N}\right)$, obtain

$$
\lim _{\epsilon \rightarrow 0}\left[\widehat{Z}_{A N} d_{A N}\left(k^{2}\right)\right]=k^{2}
$$

for arbitrary $N$, so that $\widehat{Z}_{A N}$ is the renormalisation factor and (we deal with $Q_{A}\left(k^{2}\right)$ below) the renormalised propagator is

$$
i \mathcal{D}_{A \mu \nu}^{R}=\frac{-i}{k^{2}+i \epsilon^{\prime}}\left\{g_{\mu \nu}+k_{\mu} k_{\nu} \lim _{\epsilon \rightarrow 0}\left[Q_{A}\left(k^{2}\right)\right]\right\} .
$$

To determine $Q_{W}\left(k^{2}\right)$ we could choose $c_{6}$, order by order, such that, to any given $O\left(g^{2 N}\right)$,

$$
\lim _{\epsilon \rightarrow 0}\left[\hat{\tau}_{W N L}\left(\hat{\pi}_{W N L}\right)^{-1}\right]=-\lambda^{-1}
$$

where $\hat{\tau}_{W N L}$ is defined analogously to $\hat{\pi}_{W N L}$ (see (17), (25)) and $\lambda$ is arbitrary real. Then, using (21), (34), it is easy to show that (41), for $J=W$, becomes

$$
i \mathcal{D}_{W \mu \nu}^{R}=\frac{-i}{k^{2}-\mathcal{M}_{W}^{2}+i \epsilon^{\prime}}\left[g_{\mu \nu}+(\lambda-1) \frac{k_{\mu} k_{\nu}}{k^{2}-\lambda m_{W}^{2}+i \epsilon^{\prime}}\right],
$$


which has the form of the $W$ propagator in GSW theory [7] except that the gauge parameter $\xi$ has been replaced by $\lambda$, arbitrary and independent of $\xi$ (also, $m_{W}$ in the principal denominator has been replaced by the renormalised mass $\mathcal{M}_{W}$ ). To one loop, it follows from the results given below that, with this choice of $c_{6}$, the $Z$ and $A$ propagators would also be of this form, with $\mathcal{M}_{W}, m_{W}$ replaced by $\mathcal{M}_{Z}, m_{Z} ; 0,0$.

However, we choose $\lambda=0$; more correctly, we do not impose (47) but instead take $c_{6}$ to be

$$
c_{6}=\sum c_{6(2 n)} g^{2 n} \epsilon^{-n-1}, \quad c_{6(2 n)} \neq 0 .
$$

Then (21) gives

$$
\lim _{\epsilon \rightarrow 0}\left[Q_{W, Z, A}\left(k^{2}\right)\right]=-k^{2}
$$

and, to all orders, the $W, Z$ propagators (41) become

$$
i \mathcal{D}_{J \mu \nu}^{R}(k)=\frac{-i}{k^{2}-\mathcal{M}_{J}^{2}+i \epsilon^{\prime}}\left[g_{\mu \nu}-\frac{k_{\mu} k_{\nu}}{k^{2}+i \epsilon^{\prime}}\right] .
$$

If $\rho_{A}$ is zero or less divergent than $\hat{\pi}_{A}$ at each order, then the renormalised photon propagator is also of the form (50), with $\mathcal{M}_{J}$ replaced by zero. We see below that this is so to one loop. These Landau-gauge-like propagators are independent of the arbitrary gauge parameter $\xi$.

For each ghost, the self-energy integrals are similar to those of QCD. The general self-energy integral contains the factor $(k+p)_{\mu} k_{\nu}$ coming from the terminal vertices, where $k, p$ are the ghost and a loop momentum. The self-energy is $i \tilde{\pi}=i k^{2} \tilde{\pi}_{0}\left(k^{2}\right)$, the same for every ghost $\left(\eta^{+}, \eta^{-}, \eta^{Z}, \eta^{A}\right)$. Summing the usual series gives, for each ghost, the renormalised propagator

$$
i \mathcal{D}_{\eta}^{R}\left(k^{2}\right)=-i\left[k^{2}+i \epsilon^{\prime}\right]^{-1}
$$

and the renormalisation factor

$$
\tilde{Z}_{3}=\left(1+\tilde{\pi}_{0}-\tilde{c}_{3}\right)^{-1} .
$$

It has been shown by 't Hooft $[29,30]$ that the contributions to $S$-matrix elements made by the poles at $k^{2}=0$ in the gauge boson and ghost propagators (50), (51) cancel to zero. This is the same cancellation that occurs in GSW theory between the contributions from the poles at $k^{2}=\xi m_{W}^{2}$, $k^{2}=\xi m^{2}$ in the $W, Z$ and ghost propagators.

It is possible that the renormalised propagators should be identified with physical bosons and fermions. However, such an interpretation would require 
the putative unstable-particle extension of perturbation theory suggested in section 1, and we do not pursue this question here.

We now restrict the discussion to one loop, discarding unnecessary indices. Using the propagators (12), (13), (14) we calculate the values

$$
\tau_{W}=\tau_{Z}=\tau_{A}=\tau_{A Z}=0
$$

so that, by (17),

$$
\hat{\tau}_{W}=\hat{\tau}_{Z}=\hat{\tau}_{A}=c_{6} \xi^{-1},
$$

and

$$
\rho_{A}=\rho_{A Z}=0
$$

and the divergent parts

$$
\begin{aligned}
\pi_{W \epsilon} & =\omega\left(\xi-\frac{13}{3}\right)+\frac{4}{3} \omega n \\
\pi_{Z \epsilon} & =\omega\left(\xi-\frac{13}{3}\right) \cos ^{2} \theta+\frac{4}{3} \omega n \sec ^{2} \theta\left(1-2 \sin ^{2} \theta+\frac{8}{3} \sin ^{4} \theta\right), \\
\pi_{A \epsilon} & =\omega\left(\xi-\frac{13}{3}\right) \sin ^{2} \theta+\frac{32}{9} \omega n \sin ^{2} \theta \\
\pi_{A Z \epsilon} & =\omega\left(\xi-\frac{13}{3}\right) \sin \theta \cos \theta+\frac{4}{3} \omega n \tan \theta\left(1-\frac{8}{3} \sin ^{2} \theta\right), \\
\tilde{\pi}_{\epsilon} & =k^{2} \tilde{\pi}_{0 \epsilon}=\frac{1}{2} \omega(\xi-3) k^{2}
\end{aligned}
$$

together with (4) for the divergent parts $\rho_{W \epsilon}, \rho_{Z \epsilon}$, where $\omega=g^{2}\left(16 \pi^{2} \epsilon\right)^{-1}$ and $n$ is the number of generations. Then $c_{W}, c_{Z}$ are given by (35), so that

$$
c_{W}=\omega\left[\xi+\frac{1}{3}(4 n-3)\right]-\frac{\omega}{2 m_{W}^{2}} \sum\left(m_{e j 1}^{2}+3 m_{u j 1}^{2}+3 m_{d j 1}^{2}\right),
$$

and $c_{A}, c_{B}, c_{A Z}$ are given by (9), (10).

Defining $\theta$ by

$$
m_{W}=m_{Z} \cos \theta,
$$

it follows from (17) and the results above that

$$
\begin{aligned}
\hat{\pi}_{A \epsilon} & =\hat{\pi}_{Z \epsilon}=\hat{\pi}_{W \epsilon}=\rho_{W \epsilon} m_{W}^{-2}=\rho_{Z \epsilon} m_{Z}^{-2} \\
& \equiv \hat{\pi}_{\epsilon}
\end{aligned}
$$

and that

$$
\hat{\pi}_{A Z \epsilon}=0 .
$$

These results, and so (62), are needed for the complete renormalisation to one loop of the whole theory to succeed with the BRS-invariance conditions (7) satisfied. 
With the choice (49), which at one loop is $c_{6}=c_{6(2)} g^{2} \epsilon^{-2}$, we obtain the renormalised propagator (50) for the photon (with $\mathcal{M}_{J}$ replaced by zero) as well as for $W$ and $Z$. If we had imposed (47), then (54), (63) would cause (47) to hold also for $Z$ and $A$, and the $Z$ and $A$ propagators would take the form of the $W$ propagators (48), with appropriate mass replacements.

We see from (36), (43) and (63) that $W, Z$ and $A$ lines carry the common renormalisation factor

$$
\widehat{Z}_{3}=\left(1+\hat{\pi}_{\epsilon}\right)^{-1} \text {. }
$$

On renormalising $A \leftrightarrow Z$, there are divergent $\widehat{Z}_{3}^{1 / 2}$ factors from the $A, Z$ lines multiplying the insertion $i \pi_{\mu \nu}^{A Z}$, which to one loop is finite, by (18), (64), to give zero as $\epsilon \rightarrow 0$. This simplifies diagrams and the physical interpretation of the $A$ and $Z$ propagators. In GSW theory, however, there is a double-pole $A-Z$ propagator [21].

\section{Fermion propagators and masses}

For each fermion the full propagator is given by

$$
i S_{F}^{\prime}(\not p)=i(\not p-m)^{-1}\left(1+\sigma+\sigma^{2}+\cdots\right)
$$

where

$$
\sigma=[\Sigma(\not p)-m+\kappa \not p](\not p-m)^{-1},
$$

in which $m$ stands for the initial mass $m_{1}\left(m_{e j 1}, m_{u j 1}, m_{d j 1}\right.$, zero for a neutrino), the first $m$ in $\sigma$ is from $-\mathcal{L}_{m}$ in $\mathcal{L}_{1}$, and $\kappa=\kappa_{1}+\kappa_{5} \gamma^{5}$, with, from (6),

$$
\kappa_{1}=\frac{1}{2}\left(c_{2 \ell j L}+c_{2 e j R}\right), \quad \kappa_{5}=\frac{1}{2}\left(c_{2 \ell j L}-c_{2 e j R}\right)
$$

for a charged lepton, or similar expressions for other fermions, while $\sum(\not p)$ (from the self-energy $-i \sum(\not p)$ ) is of the form

$$
\Sigma(\not p)=-a\left(p^{2}\right) \not p+b\left(p^{2}\right) .
$$

We write $a\left(p^{2}\right)=a_{1}\left(p^{2}\right)+a_{5}\left(p^{2}\right) \gamma^{5}, b\left(p^{2}\right)=b_{1}\left(p^{2}\right)+b_{5}\left(p^{2}\right) \gamma^{5}$.

To sum the series (66) in the usual way with $-m$ in the numerator of $\sigma$, we must take each $m$ to be $O\left(g^{k}\right), k>0$. We make the choice

$$
m_{e j 1}=\beta_{e j 1} g, \quad m_{u j 1}=\beta_{u j 1} g, \quad m_{d j 1}=\beta_{d j 1} g
$$


which we write generically as $m=m_{1}=\beta_{1} g$. Then, correctly to any given $O\left(g^{n}\right),(66)$ sums to

$$
\begin{aligned}
i S_{F}^{\prime}(\not p) & =i(\not p-m)^{-1}(1-\sigma)^{-1} \\
& =i[\not p-m-(\Sigma-m+\kappa \not p)]^{-1} \\
& =i\left\{\left[1+a\left(p^{2}\right)-\kappa\right] \not p-b\left(p^{2}\right)\right\}^{-1}
\end{aligned}
$$

using $A^{-1} B^{-1}=(B A)^{-1}$ and (67), (69). In (71) we see the cancellation of the $m$ terms from $\mathcal{L}_{0}, \mathcal{L}_{1}$; however, the presence of $m$ in the propagators $-i\left(\not p-m_{j 1}\right)$ is responsible for the generation of the mass term $b\left(p^{2}\right)$, from which we obtain the renormalised fermion mass.

We outline the procedure proposed for the renormalisation of the charged lepton propagators to all orders. Then we renormalise all the fermion propagators to one loop.

We renormalise each charged lepton propagator to have the pole mass

$$
\begin{aligned}
\mathcal{M} & =m_{R}-i \delta \\
& =\beta_{R} g-i\left(\delta_{2} g^{2}+\delta_{3} g^{3}+\cdots\right)
\end{aligned}
$$

where $m_{R}, \delta$ are arbitrary (to be fitted to experiment) and the generation index $j$ has been suppressed. For the electron, which is stable, the $S$-matrix reduction formula requires the renormalised and initial masses to be equal, i.e.

$$
\mathcal{M}_{e R}=m_{e R}\left(=m_{e 1 R}\right)=m_{e 1}\left(=m_{e 11}\right) .
$$

For each charged lepton we write

$$
\begin{aligned}
a\left(p^{2}\right) & =a\left(\mathcal{M}^{2}\right)+a^{\prime}\left(\mathcal{M}^{2}\right)\left(p^{2}-\mathcal{M}^{2}\right)+\frac{1}{2} ! a^{\prime \prime}\left(\mathcal{M}^{2}\right)\left(p^{2}-\mathcal{M}^{2}\right)^{2}+\cdots \\
& =a\left(\mathcal{M}^{2}\right)+2 \mathcal{M} a^{\prime}\left(\mathcal{M}^{2}\right)(\not p-\mathcal{M})+U_{a}(\not p)\left(\not p-\mathcal{M}^{2}\right)
\end{aligned}
$$

and similarly for $b\left(p^{2}\right)$, in which $U_{a}(\not p), U_{b}(\not p)$ depend on observing the order of factors shown. The denominator in (72) is then

$$
\begin{aligned}
d(\not p)= & {\left[1+a\left(\mathcal{M}^{2}\right)-\kappa\right] \mathcal{M}-b\left(\mathcal{M}^{2}\right) } \\
& +\left[1+a\left(\mathcal{M}^{2}\right)-\kappa+2 \mathcal{M}^{2} a^{\prime}\left(\mathcal{M}^{2}\right)-2 \mathcal{M} b^{\prime}\left(\mathcal{M}^{2}\right)\right](\not p-\mathcal{M}) \\
& +\left[2 \mathcal{M} a^{\prime}\left(\mathcal{M}^{2}\right)+\mathcal{M} U_{a}(\not p)-U_{b}(\not p)\right](\not p-\mathcal{M})^{2} .
\end{aligned}
$$

We make assumptions about the structure of $a\left(p^{2}\right), b\left(p^{2}\right)$ similar to those made for $\pi, \tau, \rho$ in section 3 . Writing $b\left(p^{2}\right)=\sum m_{j 1} b_{j}\left(p^{2}\right)$ and $a\left(p^{2}\right)=$ $\sum a_{2 n}\left(p^{2}\right), b_{j}\left(p^{2}\right)=\sum b_{j(2 n)}\left(p^{2}\right)$, we assume that each of $a_{2 n}\left(p^{2}\right), b_{j(2 n)}\left(p^{2}\right)$ 
is of the form (22) (with $k^{2}$ replaced by $p^{2}$ ) and that $a_{2 n, n}, b_{j(2 n, n)}$ are real and independent of $p^{2}$ and $g^{2}$. We denote $a\left(p^{2}\right), a\left(\mathcal{M}^{2}\right), b\left(p^{2}\right), b_{j}\left(p^{2}\right)$, ... taken to $N$ loops as $a_{(N)}\left(p^{2}\right), a_{(N)}\left(\mathcal{M}^{2}\right), \ldots$, and, following the treatment of $\pi, \tau, \rho$ in section 3, refer to $a_{(N)}, b_{j(N)}, b_{(N)}$ as being of $O\left(E g^{2 N}, \epsilon^{-N}\right)$, $O\left(E g^{2 N}, \epsilon^{-N}\right), O\left(E g^{2 N+1}, \epsilon^{-N}\right)$. Equation (22) holds for $a_{(N)}\left(p^{2}\right), b_{(N)}\left(p^{2}\right)$.

It is sufficient for $\kappa$ to have the form

$$
\begin{aligned}
\kappa & =\sum \kappa_{2 n} g^{2 n} \epsilon^{-n} \\
& =\sum\left(\kappa_{1(2 n)}+\kappa_{5(2 n)} \gamma^{5}\right) g^{2 n} \epsilon^{-n} .
\end{aligned}
$$

We define the leading divergent parts of $\left[a\left(p^{2}\right)-\kappa\right], b\left(p^{2}\right)$ taken to $N$ loops as

$$
\begin{aligned}
\hat{a}_{N L} & =a_{N L}-\kappa_{N} \\
& =\sum_{n=1}^{N}\left(a_{2 n, n}-\kappa_{2 n}\right) g^{2 n} \epsilon^{-n}, \\
b_{N L} & =\sum_{n=1}^{N} b_{2 n+1, n} g^{2 n+1} \epsilon^{-n},
\end{aligned}
$$

which are independent of $p^{2}$ and real, in general contain $\gamma^{5}$ parts, and are $O\left(g^{2 n} \epsilon^{-n}\right), O\left(g^{2 n+1} \epsilon^{-n}\right)$ (as well as being $\left.O\left(E g^{2 n}, \epsilon^{-n}\right), O\left(E g^{2 n+1}, \epsilon^{-n}\right)\right)$. To $N$ loops we have

$$
\begin{aligned}
{\left[a\left(p^{2}\right)-\kappa\right]_{(N)} } & =\hat{a}_{N L}+A\left(k^{2}\right)_{(2 N,-N+1)}, \\
b\left(k^{2}\right)_{(N)} & =b_{N L}+B\left(k^{2}\right)_{(2 N+1,-N+1)},
\end{aligned}
$$

where the suffices on $A, B$ indicate the orders $O\left(E g^{2 N}, \epsilon^{-N+1}\right), O\left(E g^{2 N+1}\right.$, $\left.\epsilon^{-N+1}\right)$; so that $a^{\prime}\left(k^{2}\right), a^{\prime \prime}\left(k^{2}\right), \ldots$ and $b^{\prime}\left(k^{2}\right), b^{\prime \prime}\left(k^{2}\right), \ldots$ are of these orders, respectively. We also define

$$
\widehat{Z}_{N}=\left[1+\hat{a}_{N L}\right]^{-1} \text {. }
$$

To $N$ loops, $d(\not p),(77)$, becomes, using (73), (74), (80), (81), (82), (83),

$$
\begin{aligned}
d(\not p)_{(N)}= & \left(1+\hat{a}_{N L}\right) m_{R}-\left(1+\hat{a}_{(N-1) L}\right) i \delta+O\left(E g^{2 N+2}, \epsilon^{-N}\right)+O\left(\epsilon^{-N+1}\right) \\
& -b_{N L}+\left[1+\hat{a}_{N L}+O\left(\epsilon^{-N+1}\right)\right](\not p-\mathcal{M})+\left[O\left(\epsilon^{-N+1}\right)\right](\not p-\mathcal{M})^{2},
\end{aligned}
$$


where the $O\left(\epsilon^{-N+1}\right)$ terms are of maximum order $(N-1)$ in $\epsilon^{-1}$. We impose the mass condition

$$
\hat{a}_{N L} m_{R}-b_{N L}=0
$$

for $N=1,2, \ldots$ loops. From $(80),(81),(78)$ it then follows that

$$
\kappa_{2 n}=a_{2 n, n}-m_{R}^{-1} b_{2 n, n} .
$$

At one loop $b\left(p^{2}\right)=m_{1} B\left(p^{2}\right)$, i.e. $b_{2,1}=m_{1} B_{2,1}$, so that (87) is $\kappa_{2}=$ $a_{2,1}-m_{1} m_{R}^{-1} B_{2,1}$.

From (72), the propagator is, to $N$ loops,

$$
\begin{aligned}
i S_{F(N)}(\not p) & =i\left[\widehat{Z}_{N}^{-1} \widehat{Z}_{N} d(\not p)_{(N)}\right]^{-1} \\
& =\frac{i}{\widehat{Z}_{N} d(\not p)_{(N)}} \cdot \widehat{Z}_{N}
\end{aligned}
$$

on using $(A B)^{-1}=B^{-1} A^{-1}$. From (74), (84), (85), (86), we see that, correctly to $O\left(g^{2 N+1}\right)$,

$$
\lim _{\epsilon \rightarrow 0}\left[\widehat{Z}_{N} d(\not p)_{(N)}\right]=\not p-\mathcal{M}
$$

so that, using (88) and taking the appropriate charged lepton values for $a_{2 n, n}, b_{2 n, n}$, the renormalised charged lepton propagator is

$$
i S_{F}^{R}(\not p)=i(\not p-\mathcal{M})^{-1}
$$

to all orders, since $N$ is arbitrary. From (88) we see that this propagator is multiplied on the right by the renormalisation factor $\hat{Z}_{N}$.

We now restrict the discussion to one loop and discard unnecessary indices from $a, b, \kappa$. We calculate in $d=4-2 \epsilon$ dimensions, using the propagators (12), (14), and retain only the divergent parts (writing $a_{\epsilon}=a, b_{\epsilon}=b$, $\left.\kappa_{\epsilon}=\kappa\right)$ since finite parts do not contribute, as we saw above.

For a charged lepton we obtain

$$
\begin{aligned}
a & =a_{1}+a_{5} \gamma^{5} \\
& =\frac{1}{8} \omega \xi\left[3+5 t^{2}+3\left(1-t^{2}\right) \gamma^{5}\right], \\
b & =b_{1}+b_{5} \gamma^{5} \\
& =\frac{1}{2} \omega(\xi+3) t^{2} m_{1},
\end{aligned}
$$


where $\omega=g^{2}\left(16 \pi^{2} \epsilon\right)^{-1}, t=\tan \theta$ and $m_{1}=m_{e j 1}$. We see that $b_{5}=0$. Using (79), (87) and (84) we obtain

$$
\begin{aligned}
\kappa_{1} & =\frac{1}{8} \omega \xi\left(3+5 t^{2}\right)-\frac{1}{2}\left(\frac{m_{1}}{m_{R}}\right)_{e j} \omega(\xi+3) t^{2}, \\
\kappa_{5} & =\frac{3}{8} \omega \xi\left(1-t^{2}\right), \\
\widehat{Z}_{e j} & =1-\frac{1}{2}\left(\frac{m_{1}}{m_{R}}\right)_{e j} \omega(\xi+3) t^{2}
\end{aligned}
$$

to $O\left(g^{2}\right)$, and, from $(68)$,

$$
\begin{aligned}
c_{2 \ell j L} & =\frac{1}{4} \omega \xi\left(3+t^{2}\right)-\frac{1}{2}\left(\frac{m_{1}}{m_{R}}\right)_{e j} \omega(\xi+3) t^{2}, \\
c_{2 e j R} & =\omega \xi t^{2}-\frac{1}{2}\left(\frac{m_{1}}{m_{R}}\right)_{e j} \omega(\xi+3) t^{2},
\end{aligned}
$$

where $e j$ labels the charged lepton in generation $j$. For the electron, which is stable, we must have $m_{1}=m_{R}$. It appears that we could take $m_{1}=m_{R}$ also for the higher charged leptons $(\mu, \tau, \ldots)$, which are unstable.

The renormalisation of each neutrino propagator proceeds similarly. To one loop we find that $b_{\nu}=0$, so that each neutrino must be massless. If we had placed nonzero initial neutrino mass terms $m_{\nu j 1} \bar{\nu}_{j} \nu_{j}$ in $\mathcal{L}_{m},(6)$, then $b_{\nu}$ would remain zero, neutrinos would be massless, $m_{\nu j}=0$; and then stable because of the nonzero masses of charged leptons, so that the $S$-matrix reduction formula would require $m_{\nu j}=m_{\nu j 1}$, contradicting $m_{\nu j 1} \neq 0$. At least to one loop, the theory only admits massless neutrinos. To one loop we obtain

$$
a_{\nu}=\frac{1}{8} \omega \xi\left(3+t^{2}\right)\left(1+\gamma^{5}\right)
$$

and since $\nu_{j R}$ does not appear in $\mathcal{L}$,

$$
\kappa_{\nu 1}=\kappa_{\nu 5}=\frac{1}{2} c_{2 \ell j L}
$$

with $c_{2 \ell j L}$ given by (96). From (98), (99) we find that, to $O\left(g^{2}\right)$, the renormalisation factor is

$$
\begin{aligned}
\widehat{Z}_{\nu j} & =\left[1+a_{\nu}-\kappa_{\nu}\right]^{-1} \\
& =1-\frac{1}{4}\left(\frac{m_{1}}{m_{R}}\right)_{e j} \omega t^{2}\left(1+\gamma^{5}\right) ;
\end{aligned}
$$

however, since $\widehat{Z}_{\nu j}$ stands to the right of the neutrino propagator and every neutrino vertex contains $\gamma^{\mu}\left(1-\gamma^{5}\right)$, this factor in effect is equal to $\widehat{Z}_{e j}$, so 
that to one loop we have the common lepton renormalisation factor in each generation

$$
\widehat{Z}_{2 \ell j}=1-\Delta_{\ell j}, \quad \Delta_{\ell j}=\frac{1}{2}\left(\frac{m_{1}}{m_{R}}\right)_{e j} \omega(\xi+3) \tan ^{2} \theta .
$$

In standard renormalisations of GSW theory [7,31], the right and left components of the electron propagator carry different factors $Z_{2 L}, Z_{2 R}$.

The up and down quark propagators are renormalised in the same way. To one loop we find that

$$
\begin{aligned}
& a_{u j}=\frac{1}{8} \omega \xi\left[3+\frac{17}{9} t^{2}+\left(3-\frac{5}{3} t^{2}\right) \gamma^{5}\right], \quad b_{u j}=\frac{1}{9} \omega(\xi+3) t^{2} m_{u j 1}, \\
& a_{d j}=\frac{1}{8} \omega \xi\left[3+\frac{5}{9} t^{2}+\left(3-\frac{1}{3} t^{2}\right) \gamma^{5}\right], \quad b_{d j}=-\frac{1}{18} \omega(\xi+3) t^{2} m_{d j 1} .
\end{aligned}
$$

The condition (86) then requires that

$$
\kappa_{u j 5}=a_{u j 5}, \quad \kappa_{d j 5}=a_{d j 5},
$$

leading to two expressions for $c_{2 q j L}$ :

$$
\begin{aligned}
c_{2 q j L} & =\frac{1}{4} \omega \xi\left(3+\frac{1}{9}\right) t^{2}-\frac{1}{9}\left(\frac{m_{1}}{m_{R}}\right)_{u j} \omega(\xi+3) t^{2} \\
& =\frac{1}{4} \omega \xi\left(3+\frac{1}{9}\right) t^{2}+\frac{1}{18}\left(\frac{m_{1}}{m_{R}}\right)_{d j} \omega(\xi+3) t^{2}
\end{aligned}
$$

so that

$$
\left(\frac{m_{1}}{m_{R}}\right)_{d j}=-2\left(\frac{m_{1}}{m_{R}}\right)_{u j}
$$

Then we obtain a common renormalisation factor for the up, down quarks of generation $j$, which to $O\left(g^{2}\right)$ is

$$
\widehat{Z}_{2 q j}=1-\Delta_{q j}, \quad \Delta_{q j}=\frac{1}{9}\left(\frac{m_{1}}{m_{R}}\right)_{u j} \omega(\xi+3) \tan ^{2} \theta .
$$

In addition, we obtain

$$
\begin{aligned}
c_{2 u j R} & =\frac{4}{9} \omega \xi t^{2}-\frac{1}{9}\left(\frac{m_{1}}{m_{R}}\right)_{u j} \omega(\xi+3) t^{2}, \\
c_{2 d j R} & =\frac{1}{9} \omega \xi t^{2}+\frac{1}{18}\left(\frac{m_{1}}{m_{R}}\right)_{d j} \omega(\xi+3) t^{2} .
\end{aligned}
$$

In the absence of confinement with only electroweak interactions present, a stable quark, like a stable lepton, can appear as an isolated particle in 
(in,out) states in our perturbative formalism. Assuming that the lightest quark is the (first generation) $u$ quark, $u$ is stable and the $S$-matrix reduction formula requires that $m_{u 11}=m_{u 1 R}$. Then (107) gives

$$
m_{d 11}=-2 m_{d 1 R}
$$

for the $d$ quark, so that $d$ must be unstable in this theory. (The quantisation procedure does not require that $m_{d 11}>0$.) This result is not in conflict with the instability of $d$ seen in neutron decay. Equation (107) also shows that at least one of $c, s$ and one of $t, b$ must be unstable in this theory; of course, physically they all are.

\section{Vertices}

In this section we outline a way to renormalise vertices to all orders, using the $W W Z$ vertex as an example. Then we complete the renormalisation of the whole theory to one loop.

For the $W W Z$ vertex, expressed in terms of $W^{+}, W^{-}, Z$ lines carrying incoming momenta and indices $p, \lambda ; q, \mu ; r, \nu$ respectively, the unrenormalised coupling is

$$
\begin{aligned}
v_{W W Z} & =-i g \cos \theta\left[(r-q)_{\lambda} g_{\mu \nu}+(q-p)_{\nu} g_{\lambda \mu}+(p-r)_{\mu} g_{\nu \lambda}\right] \\
& =g f_{W W Z} .
\end{aligned}
$$

All the one-loop and counterterm contributions contain the factor $v_{W W Z}$, and we shall assume that this holds true to all orders. Renormalising, and factoring out $f_{W W Z}$, we obtain the vertex quantity

$$
\begin{aligned}
G_{R} & =g\left(1+d_{1}-c_{1}\right) \widehat{Z}_{W} \widehat{Z}_{Z}^{1 / 2} \\
& =[1+O(\epsilon)] G_{R L}
\end{aligned}
$$

where $d_{1}, c_{1}$ are the vertex part and counterterm and

$$
G_{R L}=g\left(1+d_{1 L}-c_{1}\right) \widehat{Z}_{W} Z_{Z}^{1 / 2}
$$

in which $\widehat{Z}_{W}, \widehat{Z}_{Z}$ are given by (36) and

$$
d_{1 L}=\sum d_{1(2 n, n)} g^{2 n} \epsilon^{-n}
$$

is the leading divergent part of $d_{1}$ (c.f. $\pi_{N L}$ ). We have anticipated that $c_{1}$ is a series in $g^{2} \epsilon^{-1}$. 
With $d_{1 L}, c_{1}, \hat{\pi}_{W L}, \hat{\pi}_{Z L}$ correct to $O\left(g^{2 N}\right), N$ arbitrary, $G_{R L}$ is then given correctly to $O\left(g^{2 N}\right)$ by

$$
\begin{aligned}
G_{R L} & =g\left(1+d_{1 L}-c_{1}\right)\left(1-\hat{\pi}_{W L}+\hat{\pi}_{W L}^{2}-\cdots\right)\left(1-\frac{1}{2} \hat{\pi}_{Z L}+\frac{3}{8} \hat{\pi}_{Z L}^{2}-\cdots\right) \\
& =g\left[1+\sum\left(d_{1}-c_{1}\right)_{(2 n, n)} g^{2 n} \epsilon^{-n}\right]\left[1+\sum P_{2 n} g^{2 n} \epsilon^{-n}\right],
\end{aligned}
$$

where

$$
\begin{aligned}
& P_{2}=-\left(\hat{\pi}_{W L 2}+\frac{1}{2} \hat{\pi}_{Z L 2}\right) \\
& P_{4}=-\left(\hat{\pi}_{W L 4}+\frac{1}{2} \hat{\pi}_{Z L 4}\right)+\hat{\pi}_{W L 2}^{2}+\frac{3}{8} \hat{\pi}_{Z L 2}^{2}+\frac{1}{2} \hat{\pi}_{W L 2} \hat{\pi}_{Z L 2}
\end{aligned}
$$

and so on. It is clear that we can choose $c_{1}$, order by order,

$$
\begin{aligned}
& c_{1(2)}=d_{1(2,1)}+P_{2}, \\
& c_{1(4)}=d_{1(4,2)}+\left[d_{1(2,1)}-c_{1(2)}\right] P_{2}+P_{4},
\end{aligned}
$$

and so on (obtaining $\left.c_{1}=\sum c_{1(2 n)} g^{2 n} \epsilon^{-n}\right)$ such that $G_{R L}=g$ to $O\left(g^{2 N}\right), N$ arbitrary. Then, from (113), we have, in the limit $\epsilon \rightarrow 0$,

$$
G_{R}=g
$$

to all orders. We have renormalised the $W W Z$ vertex to obtain the coupling parameter $g_{R}$, with $g_{R}=g$; and with $\theta$, in $f_{W W Z}$, unchanged, i.e. we have $\theta_{R}=\theta$.

We now complete the renormalisation of the whole theory to one loop. We take $m_{1}=m_{R}$ for every charged lepton and up quark (we recall that this does not restrict the imaginary part of the mass of an unstable fermion). We obtain the standard coupling at each vertex, with $g_{R}=g, \theta_{R}=\theta$. It is convenient to drop unnecessary suffixes in what follows.

An analysis of loops shows that all boson-boson and boson-ghost vertices renormalise with $g_{R}=g, \theta_{R}=\theta$ provided that the equations

$$
\begin{aligned}
d_{1}-c_{1}-\frac{3}{2} \hat{\pi} & =0 \\
d_{5}-c_{5}-2 \hat{\pi} & =0 \\
\tilde{d}_{1}-\tilde{c}_{1}-\tilde{\pi}_{0}+\tilde{c}_{3}-\frac{1}{2} \hat{\pi} & =0
\end{aligned}
$$

hold, where $\hat{\pi}$ is given by $(63), \tilde{\pi}_{0}$ by $(60)$, and $d_{1}, d_{5}, \tilde{d}_{1}$ are reduced three-boson, four-boson and boson-ghost vertex parts, from which certain 
coupling constant and momentum-dependent terms have been factored out. By calculation and using $S U(N)$ results [28] we obtain

$$
\begin{aligned}
& d_{1}=\frac{1}{6} \omega(9 \xi-17+8 n), \\
& d_{5}=\frac{1}{3} \omega(6 \xi-4+4 n), \\
& \tilde{d}_{1}=\omega \xi
\end{aligned}
$$

Then (120), (121), (122) give

$$
\begin{aligned}
c_{1} & =\frac{1}{6} \omega(9 \xi-17+8 n)-\frac{3}{2} \hat{\pi} \\
c_{5} & =\frac{1}{3} \omega(6 \xi-4+4 n)-2 \hat{\pi} \\
\tilde{c}_{3}-\tilde{c}_{1} & =-\frac{1}{2} \omega(\xi+3)+\frac{1}{2} \hat{\pi} .
\end{aligned}
$$

The values of $\tilde{c}_{3}, \tilde{c}_{1}$ separately are not determined by renormalisation to one loop.

The renormalisation to one loop of the lepton-boson vertices is similarly straightforward. For the $e(\mu, \tau) \nu W$ vertices to renormalise to the standard coupling, we must have

$$
\frac{-i g}{2 \sqrt{2}}\left(1-\gamma^{5}\right)=\frac{-i g}{2 \sqrt{2}}\left(1-\gamma^{5}\right)\left[1-\Delta_{\ell}-\frac{1}{2} \hat{\pi}-c_{1 \ell L}+\frac{1}{4}\left(1-\tan ^{2} \theta\right) v_{1}-v_{2}\right],
$$

where the terms $v_{1}=-\omega \xi, v_{2}=-\frac{3}{2} \omega(\xi+1)$ come from the vertex parts comprising one boson and two fermion lines, and two boson and one fermion lines respectively. This gives, using (101) with $m_{1}=m_{R}$,

$$
c_{1 \ell L}=\frac{1}{4} \omega\left[\xi\left(5-\tan ^{2} \theta\right)+6\left(1-\tan ^{2} \theta\right)\right]-\frac{1}{2} \hat{\pi} .
$$

The zero $\nu \nu A$ vertices require that

$$
\begin{aligned}
c_{\ell L} & =\frac{1}{2} g \tan \theta\left(c_{1 \ell L}-v_{1}+v_{2}\right) \\
& =g \tan \theta\left\{\frac{1}{8} \omega\left[3 \xi-(\xi+6) \tan ^{2} \theta\right]-\frac{1}{4} \hat{\pi}\right\}
\end{aligned}
$$

and these values of $c_{1 \ell L}, c_{\ell L}$, independent of $j$ (generation), renormalise the $\nu \nu Z$ vertices correctly. Then the $e e Z$ (etc.) and eeA (etc.) vertices are renormalised correctly on choosing

$$
c_{e R}=\frac{1}{2} g \tan \theta\left[\omega(\xi-3) \tan ^{2} \theta-\hat{\pi}\right] .
$$


The renormalisation of quark vertices goes through similarly. We obtain, taking $m_{1}=m_{R}$ for each up quark, the generation-independent results that

$$
\begin{aligned}
c_{1 q L} & =\frac{1}{4}\left(1-\frac{1}{9} \tan ^{2} \theta\right) v_{1}-v_{2}-\Delta_{q}-\frac{1}{2} \hat{\pi}, \\
& =\frac{1}{4} \omega\left[5 \xi+6-\frac{1}{3}(\xi+4) \tan ^{2} \theta\right]-\frac{1}{2} \hat{\pi}, \\
c_{q L} & =g \tan \theta\left\{\frac{1}{12} \hat{\pi}-\frac{1}{8} \omega\left[\xi\left(1-\frac{1}{9} \tan ^{2} \theta\right)-\frac{4}{9} \tan ^{2} \theta\right]\right\}, \\
c_{u R} & =g \tan \theta\left\{\frac{1}{3} \hat{\pi}-\frac{2}{9} \omega(\xi-1) \tan ^{2} \theta\right\}, \\
c_{d R} & =g \tan \theta\left[-\frac{1}{6} \hat{\pi}-\frac{1}{9} \omega \tan ^{2} \theta\right] .
\end{aligned}
$$

To one loop the $S U(2)_{L} \times U(1)_{Y}$-invariance conditions (7) are

$$
\begin{aligned}
c_{W}-c_{1} & =c_{1}-c_{5} \\
& =\tilde{c}_{3}-\tilde{c}_{1} \\
& =c_{2 \ell j L}-c_{1 \ell j L} \\
& =c_{2 q j L}-c_{1 q j L},
\end{aligned}
$$

where, with $m_{1}=m_{R}$ for the charged leptons and up quarks, the $c_{. . j L}$ are independent of the generation index $j$. We see that these equations are satisfied by the values given by (61), (128), (129), (130), (96), (132), (105) and (135).

The renormalisation of the theory to one loop is complete, with the condition (7) satisfied, and with the bosons and fermions possessing appropriate real and complex masses.

Renormalised to one loop, the $W, Z$, photon, ghost and fermion propagators, and the coupling parameters $g, \theta$, are independent of the gauge parameter $\xi$. Then the resulting $S$-matrix elements are independent of $\xi$.

\section{Conclusions and discussion}

We have set up a perturbative $S U(2)_{L} \times U(1)_{Y}$ electroweak theory containing $W, Z$, photon, ghost, lepton and quark fields, but no Higgs field. We

make an unconventional choice for the unperturbed Lagrangian $\mathcal{L}_{0}$. Then physical $W, Z$ and fermion masses are obtained on renormalisation to one loop by a somewhat unorthodox but systematic method. The renormalisation preserves the usual couplings, with renormalised parameters $g_{R}, \theta_{R}$ equal to the original parameters $g, \theta$, requires neutrinos to be massless and $m_{W}=m_{Z} \cos \theta$ to hold, and causes the $A \leftrightarrow Z$ mixing to drop out. Also, the theory renormalised to one loop gives $S$-matrix elements that are independent of the gauge parameter $\xi$. 
The theory should be renormalisable to all orders, as we have outlined such a systematic procedure to renormalise the boson and fermion propagators and the vertices, to arbitrary order. As discussed in section 1, a treatment of the unitarity of this theory, which contains unstable particles (and similarly a proper treatment of unitarity in the Standard Model), waits for an extension of perturbation theory that incorporates unstable particles consistently.

The choice of Dirac, not Majorana, masses (2) for the fermions forced the neutrinos to be massless, as neutrinos lack right-handed partners in the Standard Model. In turn, this choice implied a charge-neutral vacuum, unbroken lepton number and QED gauge symmetries, and a massless photon (55). Furthermore, careful consideration of the $S U(2)_{L}$ symmetry properties of the $\hat{\pi}_{J}$ shows that the phenomenologically necessary relation (62) between $m_{W}$ and $m_{Z}$ is automatic. Majorana fermion masses would violate this requirement, but they should be explored as a theoretical possibility.

The mechanism of fermion and gauge boson mass generation (dynamically broken chiral and gauge symmetries) presented here and earlier [3] bears similarities to existing models, but with some important differences. The fermions breaking chiral and gauge symmetries are the Standard Model fermions themselves, with no new particles or interactions necessary, unlike technicolour [32] and top condensate [33] models. And our scheme is perturbative in the gauge coupling. This feature differs from the non-perturbative "soft" fermion self-energy ansatz for dynamically breaking chiral gauge symmetries discovered by Jackiw and Johnson [34] and applied to the Standard Model by Carpenter et al. [35]. In that scenario, the gauge boson and fermion mass(es) are linked by a finite relationship after renormalisation that predicts too large a top quark mass (or equivalently, too small $W$ and $Z$ masses). Our scheme renormalises the fermion and gauge boson masses separately, so that their masses remain independent.

On the other hand, simple Ward identity arguments [36] imply in all of these models an effective Higgs-like scalar resonance with mass $m_{H} \simeq$ $\sqrt{2} m_{t} \simeq 250 \mathrm{GeV}$, independent of model solution details. The question of choosing the perturbative or non-perturbative solution can only be settled by explicit calculation of the vacuum energy, an issue, like unitarity and Majorana masses, we leave to a subsequent treatment. 


\section{Acknowledgments and Note}

D.C.K. thanks V. A. Miransky of the Bogolyubov Institute for Theoretical Physics, Kiev, for helpful discussions and Betty Nicholson and Ann Milligan for their help with completing the manuscript.

This paper is dedicated to the memory of Angus Nicholson (1927-1999). A native of Australia and receiving his M.Sc. and Ph.D. degrees in physics at London and Birmingham Universities, he spent much of his working life as research scientist with the Australian Defence Science and Technology Organisation. After his retirement, he continued theoretical physics work as visiting scholar at The Australian National University, Canberra, until his death.

\section{References}

[1] W.J. Marciano, "Status of the Standard Model", in Proc. Conf. on Intersections between Particle and Nuclear Physics, St Petersburg, Florida, June 1994 (New York: A.I.P., 1995) 3.

[2] R.S. Chivikula, B.A. Dobrescu and E.H. Simmons, Phys. Lett. B 401, 74 (1997).

[3] A.F. Nicholson and D.C. Kennedy, UF-IFT-HEP-96-29, LANL hep-th/9702113.

[4] J. Schwinger, Phys. Rev. 128, 2425 (1962).

[5] S. Coleman, R. Jackiw and L. Susskind, Ann. Phys. (NY) 93, 267 (1975).

[6] K. Aoki, Z. Hioki, R. Kawabe, M. Konuma and T. Muta, Supp. Prog. Theor. Phys. 73, 1 (1982).

[7] D. Bailin and A. Love, Introduction to Gauge Field Theory (Bristol, UK: IOP Publishing, 1993).

[8] J.C. Collins, Renormalization (Cambridge: Cambridge University Press, 1984).

[9] A.J. Buras and P.H. Weisz, Nucl. Phys. B 333, 66 (1990).

[10] S.A. Larin, Phys. Lett. B 303, 113 (1993). 
[11] L. Avdeev, J. Fleischer, S. Mikhailov and O. Tarasov, Phys. Lett. B 336, 560 (1994); 349, 597 (E) (1995).

[12] K.G. Chetyrkin, J.H. Kühn and M. Steinhauser, Phys. Rev. Lett. 19, 3394 (1995).

[13] T.L. Trueman, Z. Phys. C 69, 525 (1996).

[14] K.G. Chetyrkin and A. Kwiatkowski, Nucl. Phys. B 461, 3 (1996).

[15] M. Chanowitz, M. Furman and I. Hinchliffe, Nucl. Phys. B 159, 225 (1979).

[16] J.D. Bjorken and S.D. Drell, Relativistic Quantum Fields (New York: McGraw-Hill, 1965).

[17] A. Martin, "Unstable Particles", in Proc. NATO Advanced Study Inst. on $Z^{0}$ Physics, Cargèse, France, 1990, eds. M. Lévy et al. (New York: Plenum Press, 1991) 483.

[18] C. Bernardini, L. Maiani and M. Testa, Phys. Rev. Lett. 71, 2687 (1993).

[19] J.-P. Marchand, Foundations of Physics 27, 215 (1997).

[20] G.G. Ross, Grand Unified Theories (Menlo Park, CA: Benjamin/Cummings, 1984).

P. Becher, M. Böhm and H. Joos, Gauge Theories of Strong and Electroweak Interactions (Chichester, UK: Wiley, 1984).

[21] F.A. Berends, "Electroweak Radiative Corrections for $Z$ Physics", in Proc. NATO Advanced Study Inst. on $Z^{0}$ Physics, Cargèse, France, 1990, eds. M. Lévy et al. (New York: Plenum Press, 1991) 321.

[22] A. Sirlin, Phys. Rev. Lett. 67, 2127 (1991); Phys. Lett. B267, 240 (1991).

[23] In the LSZ formalism: Ref. 23; C. Itzykson and J-B. Zuber, Quantum Field Theory (New York: McGraw-Hill, 1980).

[24] C. Itzykson and J.-B. Zuber, Quantum Field Theory (New York: McGraw-Hill, 1980).

[25] J. Papavassiliou and A. Pilaftsis, Phys. Rev. Lett. 75, 3060 (1995). 
[26] K. Philippides and A. Sirlin, Phys. Lett. B 367, 377 (1996).

[27] M. Passera and A. Sirlin, Phys. Rev. Lett. 77, 4146 (1996).

[28] P. Pascual and R. Tarrach, QCD: Renormalization for the Practitioner, Lecture Notes in Physics 194 (Berlin: Springer-Verlag, 1984).

[29] G. 't Hooft, Nucl. Phys. B 33, 173 (1971).

[30] G. 't Hooft, Nucl. Phys. B 35, 167 (1971).

[31] J.P. Cole, Progr. Part. Nucl. Phys. 12, 241 (1984).

[32] S. Weinberg, Phys. Rev. D 19, 1277 (1979); L. Susskind, Phys. Rev. D 20, 2619 (1979).

[33] V.A. Miransky, Mod. Phys. Lett. A 4, 1043 (1989); W.J. Marciano, Phys. Rev. D 41, 219 (1990); W.A. Bardeen, C.T. Hill and M. Lindner, Phys. Rev. D 41, 1647 (1990).

[34] R. Jackiw and K. Johnson, Phys. Rev. D 8, 2386 (1973).

[35] J. Carpenter, R. Norton, S. Siegemund-Broka and A. Soni, Phys. Rev. Lett. 65, 153 (1990).

[36] K. Shen and Y.-P. Kuang, Phys. Rev. D 57, 6386 (1998). 